\title{
Primer registro de Agrostichthys parkeri en el Océano Pacífico suroriental (Osteichthyes: Regalecidae)
}

First record in the southeastern Pacific Ocean of Agrostichthys parkeri

(Osteichthyes: Regalecidae)

\section{Germán Pequeño ${ }^{1}$}

${ }^{1}$ Instituto de Zoología ‘Ernst F. Kilian’, Universidad Austral de Chile, Casilla 567, Valdivia, Chile. gpequeno@uach.cl

\begin{abstract}
The discovery of one specimen of Agrostichthys parkeri in Chilean waters is reported. This is the first record of this fish species for the southeastern Pacific Ocean. The specimen, $1.5 \mathrm{~m}$ total length, was found in shallow waters at $2 \mathrm{~m}$ depth, in southern Chiloé Island, Chile. The number of species belonging to the order Lampridiformes in Chile is increased to nine.
\end{abstract}

Key words: Ichthyogeography, Lampridiformes, bony fishes, Chile

\section{INTRODUCCIÓN}

La región de los fiordos australes chilenos requiere de urgentes estudios taxonómicos, ecológicos y biogeográficos. Según Gallardo (2009) 'Con menos de un 0,0075\% de la superficie, con algún tipo de protección sobre el papel, la región de los fiordos de Chile debe ser una de las zonas marinas peor conservadas del mundo'; más aún, la fauna, especialmente la ictiofauna, es muy poco conocida (i.e., Jenyns 1842, Lönnberg 1907).

El orden Lampridiformes ha sido estudiado en forma parcial en Chile debido a que diversos autores han analizado algunos de sus taxa por separado (Nakamura 1986, Pequeño 1991, Cortés et al. 1995). La familia Regalecidae, es una de las cuatro familias que integran este orden en la zona y ha sido conocida solamente por la presencia de Regalecus glesne Ascanius, 1772. Las otras familias son Lamprididae, Stylephoridae y Trachipteridae.

El orden Lampridiformes es comparativamente menos conocido que otros grupos de peces en Chile; especialmente la familia Regalecidae constituye en cierto modo una agrupación rara, tanto para los habitantes de la región, como para los especialistas en la materia. Por eso, es necesario y conveniente, comunicar hallazgos como el presente, que además de su importancia para la taxonomía y sistemática de la especie, contribuyen a conocer mejor sus aspectos etológicos, ecológicos, biológicos generales y biogeográficos. Se informa un registro nuevo de una especie del orden Lampridiformes en Chile, en particular de la familia Regalecidae, con la connotación de haberse encontrado en la zona de los canales australes de Chile.

\section{Materiales y MÉTOdos}

Se obtuvo un espécimen de Agrostichthys parkeri (Benham, 1904) capturado en Auchac Chiloé, sur de Chile (ca., $43^{\circ} 01^{\prime} 49^{\prime \prime}$, $73^{\circ} 32^{\prime} 57^{\prime \prime} \mathrm{W}$ ), a $2 \mathrm{~m}$ de profundidad y que midió $150 \mathrm{~cm}$ de longitud total. El reconocimiento taxonómico se hizo mediante el uso de literatura especializada (Trunov 1982, Heemstra 1986, Paulin et al. 1989, Trunov \& Kukuev 2005, Ruíz \& Gosztonyi 2010). El ejemplar no fue preservado. Fotografías en color del ejemplar se guardaron en el Instituto de Zoología de la Universidad Austral de Chile (UACH) ${ }^{1}$. Las medidas fueron determinadas en $\mathrm{cm}$ y mm. Las abreviaturas en el texto son LT = longitud total, $\mathrm{LC}=$ longitud de la cabeza, $\mathrm{DOH}=$ diámetro ocular horizontal, AC = altura máxima del cuerpo.

\section{RESULTADOS Y DISCUSIÓN}

El ejemplar de $150 \mathrm{~cm}$ LT capturado en Auchac, luego de consultar literatura especializada (Pequeño 1989, 1997, Chirichigno \& Vélez 1998, Pequeño 2009), resultó ser desconocido en el ámbito de la ictiología de Chile y también de costas adyacentes. Sin embargo, la revisión de textos de África del Sur y Nueva Zelandia (Trunov 1982, Heemstra 1986, Paulin et al. 1989), permitió comparar un pez con

\footnotetext{
${ }^{1}$ Contactar al autor de este estudio
} 
características semejantes a las de nuestro hallazgo de Chiloé. El ejemplar obtenido se describe como extremadamente largo, en comparación con la altura de su cuerpo, la cual está contenida 32 veces en la LT. La cabeza está contenida 11,8 veces en la LT. El diámetro ocular (DOH) está contenido 3,8 veces en la LC (Fig. 1). Presenta dos características, que juntas, solo se dan en una especie conocida del orden Lampridiformes, las cuales son: los radios muy largos con los que se inicia la aleta dorsal (en este ejemplar quebrados, alcanzan aproximadamente dos veces la LC, mientras que en organismos completos alcanzan aproximadamente 3,3 veces la LC) y una premaxila que se extiende hacia delante, a lo largo de lo que empieza a conformar el perfil dorsal de la cabeza. También la extremidad caudal dividida en dos a modo de una tijera, conforma un conjunto único, que solamente está representado en la especie Agrostichthys parkeri (Fig. 2), que fuera originalmente descrita como Regalecus parkeri Benham, 1904, para aguas de Nueva Zelandia. El color es, en general, plateado azulado, destacándose el color prácticamente negro de las aletas medianas. El ejemplar coincide con la reciente descripción de especímenes del Atlántico suroccidental, frente a Argentina (Trunov \& Kukuev 2005, Ruíz \& Gosztonyi 2010).

Los peces del orden Lampridiformes son considerados entre las familias propias del epipélagos y del mesopélagos. Sin embargo, por causas no bien conocidas, hay casos en los cuales determinados especímenes se acercan a la costa y pueden ser capturados muy cerca de la orilla y a poca profundidad. Este es el caso del ejemplar registrado en Auchac, cerca de Quellón en la parte sur de la Isla Grande de Chiloé. El ejemplar no requiere mayor análisis desde el punto de vista morfológico, pues coincide bien con los antecedentes encontrados en la literatura (Trunov 1982, Heemstra 1986, Paulin et al. 1989, Trunov \& Kukuev 2005). En cuanto a su significado ictiogeográfico, resulta ser una evidencia más para apoyar la condición circumpolar de la especie (Trunov \& Kukuev 2005, Eschmeyer 2010), si bien es cierto la especie contaba con registros en varias partes del hemisferio sur, aún no había registros documentados del sector suroriental del Océano Pacífico. En el hemisferio sur,

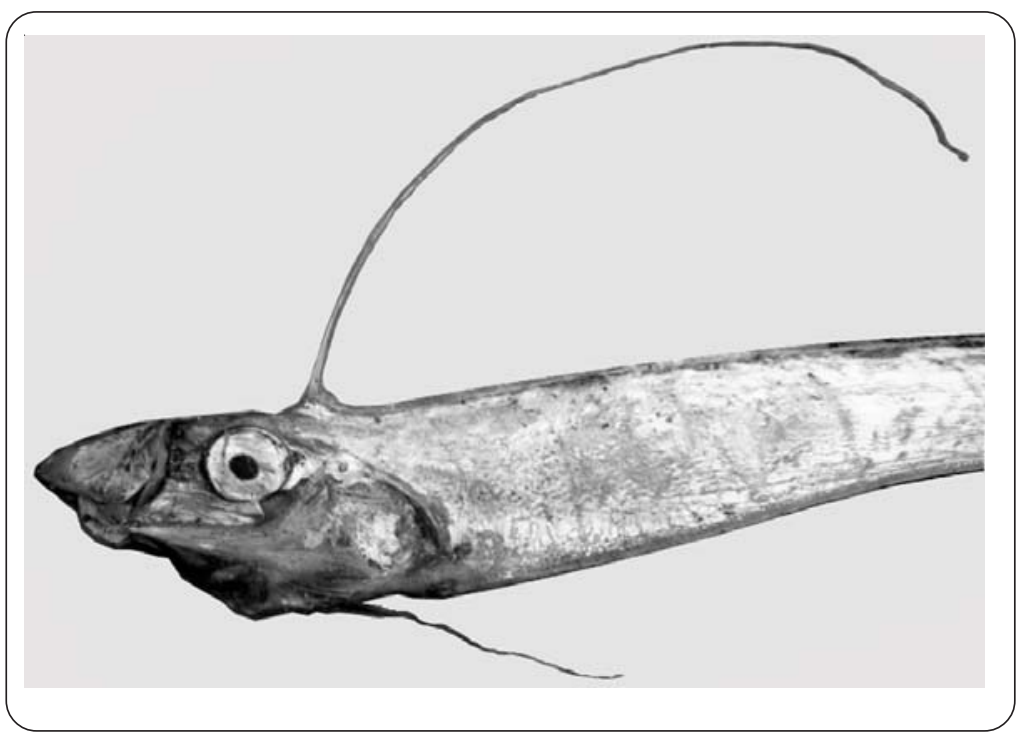

Figura 1. Sector anterior del cuerpo del ejemplar de Agrostichthys parkeri capturado en Auchac, Chiloé / Anterior part of the body of Agrostichthys parkeri captured in Auchac, Chiloé

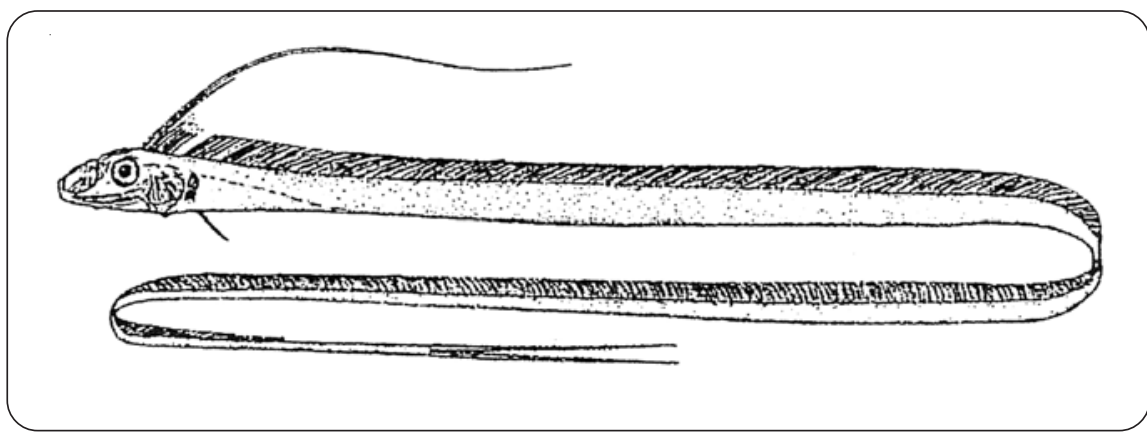

Figura 2. Esquema representativo de Agrostichthys parkeri (Modificado de Heemstra 1986) / Representative sketch of Agrostichthys parkeri (Modified from Heemstra 1986). 


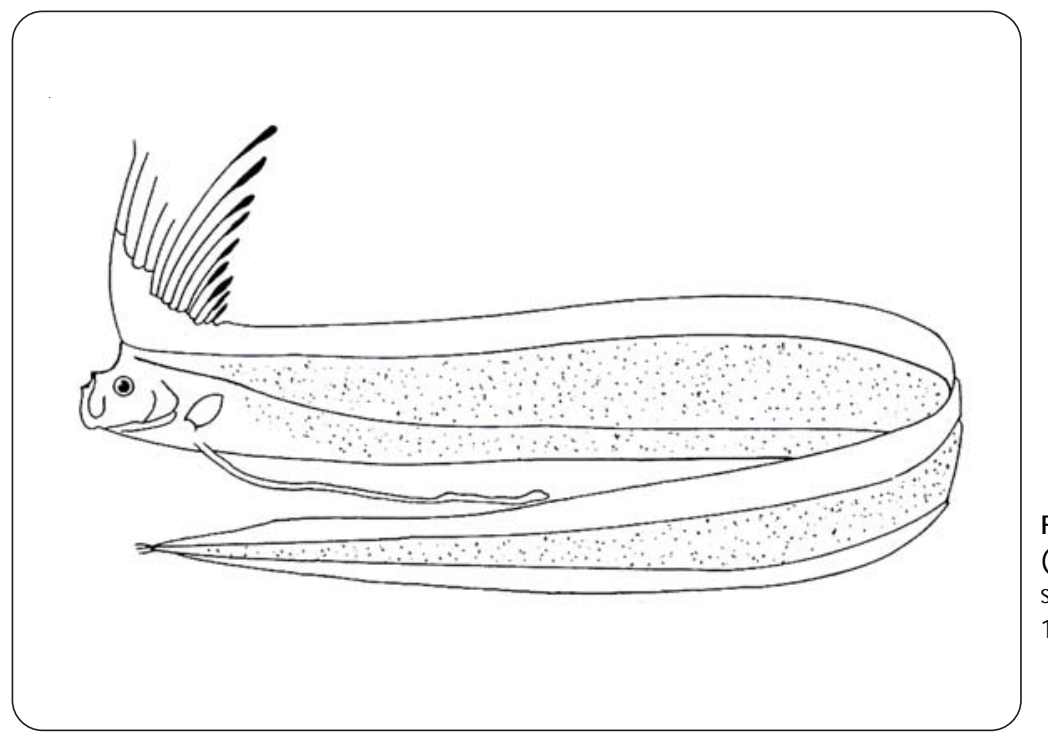

Figura 3. Esquema representativo de Regalecus glesne (Modificado de Paulin et al. 1989) / Representative sketch of Regalecus glesne (Modified from Paulin et al. 1989)

A. parkeri ha sido registrada en Nueva Zelandia y aguas adyacentes, Australia, África del sur y mares de Argentina (Trunov 1982, Heemstra 1986, Paulin et al. 1989, Paxton et al. 1989, Trunov \& Kukuev 2005, Ruíz \& Gosztonyi 2010). Hay también antecedentes para el hemisferio norte (CastroAguirre et al. 1991, Galván-Magaña et al. 1999), pero concordamos con Trunov \& Kukuev (2005), en la necesidad de revisar la taxonomía del género a nivel global.

Desde el punto de vista taxonómico, la primera especie de la familia Regalecidae registrada en Chile fue Regalecus glesne (Bahamonde \& Pequeño 1975) (Fig. 3), siendo Agrostichthys parkeri, la segunda especie de esta familia registrada en Chile descrita en el presente estudio. Por lo tanto, de acuerdo con los datos existentes (Nakamura 1986, Pequeño 1989, 1991, Sielfeld et al. 1995, Pequeño 1997) el orden Lampridiformes en aguas chilenas alcanza ahora a nueve especies, con la siguiente ordenación taxonómica:

Familia Lamprididae

Lampris regius (Bonnaterre, 1788)

Familia Regalecidae

Agrostichthys parkeri (Benham, 1904)

Regalecus glesne Ascanius, 1772

Familia Stylephoridae

Stylephorus chordatus Shaw, 1791

Familia Trachipteridae

Desmodema polysticta (Ogilby, 1897)

Trachipterus altivelis (Ogilby, 1897)

Trachipterus fukusakii Fitch, 1964

Trachipterus trachipterus (Gmelin, 1789)

Zu cristatus (Bonelli, 1820).
Particularmente la familia Regalecidae, es representada por dos taxa, tanto para Chile como Nueva Zelandia. Modificando una clave de Paulin et al. (1989), estas dos especies se pueden diferenciar de la siguiente manera:

- Entre 40 y 58 branquispinas; premaxila en ángulo recto respecto del perfil frontal; axis de la maxila vertical, los primeros 14 radios dorsales alargados como penacho .Regalecus glesne (Fig. 3) - Entre 8 y 10 branquispinas, premaxila se extiende hacia delante a lo largo del perfil dorsal de la cabeza; axis de la maxila oblicuo; sólo los dos primeros radios dorsales son muy alargados, sin aspecto de penacho........... .Agrostichthys parkeri (Figs. 1, 2)

Este registro contribuye al conocimiento de la diversidad ictiofaunística del Pacífico suroriental y en particular de los canales australes de Chile, en concordancia con las necesidades planteadas por Gallardo (2009). Con esto, se indica la necesidad de revisión del orden Lampridiformes en la región sur de Chile.

\section{Agradecimientos}

Se agradece la obtención de datos en terreno por parte de Diego Rivera (Terra) y de Jorge Pacheco. A Héctor Flores (UCN, Coquimbo, Chile) por su contribución en la búsqueda de material bibliográfico. Richard L. Haedrich (Memorial University, Newfoundland, Canada) y M. Eric Anderson (South African Institute for Aquatic Biodiversity, Grahamstown, South Africa) por la donación de valiosas colecciones bibliográficas. Valeria Pérez (Universidad 
Austral de Chile, Valdivia) por su colaboración en el montaje fotográfico. Estos son resultados parciales del Proyecto DID2005-03 de la Universidad Austral de Chile.

\section{LITERATURA CITADA}

Bahamonde N \& G Pequeño. 1975. Peces de Chile. Lista Sistemática. Museo Nacional de Historia Natural, Chile, Publicación Ocasional 21: 1-20.

Benham WB. 1904. An apparently new species of Regalecus (R. parkeri). Transactions of the New Zealand Institute 36(23): 198-200. [Pl. 9]

Castro-Aguirre JL, J Arvizu-Martínez \& C AlarcónGonzález. 1991. Una especie nueva de Regalecus (Pisces. Lampridiformes) hallada en la Bahía de La Paz, Baja California Sur, México, con notas y observaciones taxonómicas y biogeográficas de la familia Regalecidae. Anales de la Escuela de Ciencias Biológicas, México 34: 159-171.

Chirichigno N \& J Vélez. 1998. Clave para identificar los peces marinos del Perú, 496 pp. Publicación Especial, Instituto del Mar del Perú, Callao.

Cortés N, M Arriaza \& C Oyarzún. 1995. Nuevos registros de Trachipterus trachypterus (Gmelin, 1789) para el Pacífico suroriental, con una revisión de ejemplares congenéricos de Chile (Osteichthyes, Trachipteridae). Revista de Biología Marina 30(2): 265-273.

Eschmeyer WN. 2010. Catalog of fishes electronic version. [en línea] <http://research.calacademy.org/ichthyology/ catalog/fishcatmain.asp>

Gallardo VA. 2009. Prefaces. En: Haussermann V \& G Försterra (eds). Marine benthic fauna of Chilean Patagonia, p. 9. Nature in Focus, Santiago de Chile.

Galván-Magaña F, LA Abitia-Cárdenas \& FJ GutiérrezSánchez. 1999. Records of the oarfish Regalecus glesne Ascanius, 1772 in the eastern Pacific Ocean. Bulletin of the Southern California Academy of Sciences 98(3): 127130 .

Heemstra PC. 1986. Family $N^{\circ} 222$, Regalecidae. En: Smith MM \& PC Heemstra (eds). Smith’s Sea Fishes, pp. 11047, CTP Book Printers, Cape Town.
Jenyns L. 1842. Fish. En: Darwin C (ed). Voyage of HMS Beagle during the years 1832-1836, pp. 1-172. C Darwin, London.

Lönnberg E. 1907. Fische. Ergebnisse der Hamburger Magalahenischen Sammelreise 1892-93(1): 1-16.

Nakamura I. 1986. Important fishes trawled off Patagonia, 369 pp. Japan Marine Fishery Resource Research Center, Tokyo.

Paulin C, C Roberts, A Stewart \& P McMillan. 1989. New Zealand fish: A complete guide. National Museum of New Zealand Miscellaneous Series 19: 1-279.

Paxton J, DF Hoese, GR Allen \& JE Hanlei. 1989. Zoological catalogue of Australia 7, Pisces, Petromyzontidae to Carangidae, 665 pp. Australian Government Public Services, Canberra.

Pequeño G. 1989. Peces de Chile. Lista sistemática revisada y comentada. Revista de Biología Marina 24(2): 1-132.

Pequeño G. 1991. Nuevos registros de Trachipterus trachipterus (Gmelin, 1789) frente a Valdivia (Osteichthyes, Trachipteridae). Boletín de la Sociedad de Biología de Concepción, Chile 62: 119-122.

Pequeño G. 1997. Peces de Chile. Lista sistemática revisada y comentada: Addendum. Revista de Biología Marina y Oceanografía 32(2): 77-94.

Pequeño G. 2009. Gnathostomata \& Petromyzontida - Fishes, En: Hausserman V \& G. Försterra (eds). Marine benthic fauna of Chilean Patagonia, pp. 907-947. Nature in Focus, Santiago de Chile.

Ruíz AE \& A Gosztonyi. 2010. Records of regalecid fishes in Argentine waters. Zootaxa 2509: 62-66.

Sielfeld W, M Vargas \& R Fuenzalida. 1995. Peces mesopelágicos frente a la costa norte de Chile $\left(18^{\circ} 25^{\prime}\right.$ 214’'S). Investigaciones Marinas 23: 87-97.

Trunov IA. 1982. Species of the family Regalecidae (Lampridiformes) from the southeastern Atlantic ocean. Journal of Ichthyology 22(1): 1-6. [2 fig].

Trunov IA \& EI Kukuev. 2005. New data on fish of the family Trachipteridae and Regalecidae (Trachipteroidei) from the Argentina Trench (Southwestern Atlantic). Journal of Ichthyology 45(3): 223-228.

Recibido el 10 de septiembre de 2010 y aceptado el 29 de octubre de 2010 